\title{
Research on Sliding Mode Control for Steer-by-Wire System in Forklift
}

\author{
Jun-Jie HUANG ${ }^{1}$, Ben-Xian XIAO ${ }^{2}$, Yang LIU ${ }^{3}$, Yi-Gang $\mathrm{HE}^{4}$ \\ ${ }^{1}$ Institute of Industry \& Equipment Technology, School of Electrical Engineering and Automation, Hefei University of Technology, Hefei, \\ P.R.China \\ ${ }^{2}$ School of Electrical Engineering and Automation, Hefei University of Technology, Hefei, P.R. China \\ ${ }^{3}$ Institute of Industry \& Equipment Technology, Hefei University of Technology, Hefei, P.R. China \\ ${ }^{4}$ School of Electrical Engineering and Automation, Hefei University of Technology, Hefei, P.R. China

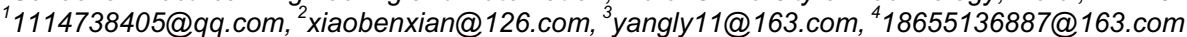

\begin{abstract}
Aiming at steering stability and wheel angle tracking of steer-by-wire (SBW) three wheeled forklift, steering dynamic model and SBW system mathematical model of three wheeled forklift are established. A control strategy for the ideal transmission ratio is introduced based on this model, which ensures forklift steering gain invariant. A sliding mode controller can then be designed based on the bound information of uncertain system parameters, uncertain self-aligning torque, and external disturbances. The results of simulation show the control strategies above can effectively reduce the sideslip angle when the forklift is steering and improve the sensitivity and stability of the steering forklift; at the same time can effectively restrain the parameter perturbation of internal system and external disturbance, which improves the tracking performance of the wheel angle.
\end{abstract}

\section{Introduction}

The forklift usually works in small working space to handle cargo with low speed, so more need to ensure the steering maneuverability and handling stability ${ }^{[1]}$. The three wheeled forklift has the characteristic of small size, it uses advanced dual-front technology and rear wheel active steering technology, can achieve 90-degree steering, has advantage of small turning radius, more effort to operate, so very suitable for working in narrow space.

Conventional forklifts, which are mechanical steering, can't take the initiative to control the angular transmission characteristics and force transfer characteristics of the steering system. The Steer-by-Wire (SBW) system eliminates the mechanical connection between steering handle and steering wheels in a conventional steering system and transmits the control signals via electrical signals, so that can be freely designed[2-5].

Nowadays, the research on the vehicle steering system is mostly based on the variable transmission ratio and the strategy of yaw stability control, and does not take into account the tracking performance of the wheel angle of SBW system. In this paper, for the three wheeled forklift equipped with SBW system, the stability of the steering forklift and the problem of wheel angle tracking are considered. Based on the ideal transmission ratio control which the yaw rate gain is fixed, sliding mode angle tracking controller based on variable parameters is designed. Through simulation experiments, it is verified that the proposed scheme can improve the stability of the steering forklift and the tracking performance of wheel angle.

\section{Modeling of SBW System for Three Wheeled Forklift}

In this paper, a three wheel steering model with $2 \mathrm{DOF}$ (which are lateral and yaw) was established.

\subsection{Three Wheeled Forklift Steering Dynamics Model Based on Ground Coordinate System}

As shown in Fig.1, $O^{\prime}$ is the steering center of the forklift, $O$ is the centroid of forklift. The parameters of rear wheel steering dynamic model of three wheeled forklift are shown as follows: $u$ is the longitudinal velocity of the vehicle centroid $(\mathrm{m} / \mathrm{s}), v$ is the lateral velocity of the vehicle centroid $(\mathrm{m} / \mathrm{s}), \beta$ is the sideslip angle at the centroid (rad). $\omega_{r}$ is the yaw $\mathrm{rate}(\mathrm{rad} / \mathrm{s})$, $\delta$ is the angle of steering rear wheel(rad), $\alpha_{1}$ and $\alpha_{2}$ are the sideslip angle of left front wheel and right front wheel respectively( $\mathrm{rad}), \alpha_{3}$ is the sideslip angle of rear wheel (rad), $F_{y 1}$ and $F_{y 2}$ are the lateral force of left front wheel and right front wheel respectively(N), $F_{y 3}$ is the lateral force of rear wheel $(\mathrm{N}), a$ and $b$ are the distance from centroid to front axle and rear axle 
respectively $(\mathrm{m}), \mathrm{L}$ is the distance from front wheel center to rear wheel center(m), $\mathrm{c}$ is the center distance between the left front wheel and the right front wheel(m), $M$ is the mass of the whole forklift $(\mathrm{kg})$.

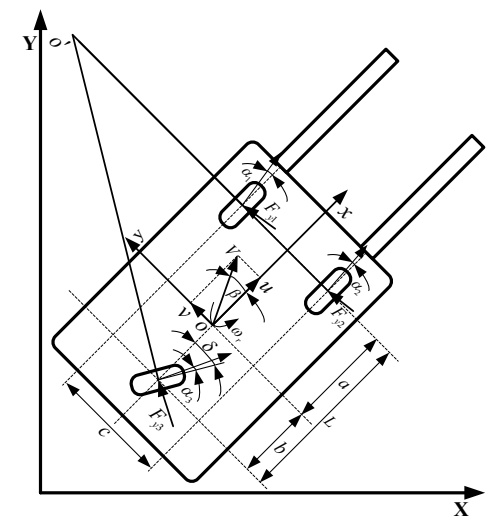

Figure 1. Dynamic model of rear wheel steering of forklift

According to the Fig.1, the resultant force along the $y$-axis and the torque around the centroid when the forklift is steering are shown below:

$$
\left\{\begin{array}{l}
M u\left(\dot{\beta}+\omega_{r}\right)=F_{y 1}+F_{y 2}+F_{y 3} \cos \delta \\
J_{z} \dot{\omega}_{r}=-a F_{y 1}-a F_{y 2}+b F_{y 3} \cos \delta
\end{array}\right.
$$

Where, the sideslip angle at the centroid $\beta \approx \tan \beta=v / u$. When a forklift is steering, the transformation of its rear wheel angle is slow, so in the moment of steering, it can be considered that $\cos \delta \approx 1 . J_{z}$ is the moment of inertia of the vehicle around the $\mathrm{z}$-axis $\left(\mathrm{kg} \cdot \mathrm{m}^{2}\right.$ ), so (1) can be written as:

$$
\left\{\begin{array}{l}
M u\left(\dot{\beta}+\omega_{r}\right)=M\left(\dot{v}+u \omega_{r}\right)=F_{y 1}+F_{y 2}+F_{y 3} \\
J_{z} \dot{\omega}_{r}=-a F_{y 1}-a F_{y 2}+b F_{y 3}
\end{array}\right.
$$

When the sideslip angle is less than $5^{\circ}$, the lateral force of the tire is linearly related to the sideslip angle[4], so the lateral force of three wheels are:

$$
F_{y 1}=-C_{1} \alpha_{1}, F_{y 2}=-C_{2} \alpha_{2}, F_{y 3}=-C_{3} \alpha_{3}
$$

Where, $C_{1}$ and $C_{2}$ are the cornering stiffness of left front wheel and right front wheel, $C_{3}$ is the cornering stiffness of rear wheel.

According to the relative motion principle:

$$
\left\{\begin{array}{l}
\alpha_{1}=\frac{v-a \omega_{r}}{u+\frac{c}{2} \omega_{r}} \approx \beta-\frac{a \omega_{r}}{u}, \alpha_{2}=\frac{v-a \omega_{r}}{u-\frac{c}{2} \omega_{r}} \approx \beta-\frac{a \omega_{r}}{u} \\
\alpha_{3}=\frac{v+b \omega_{r}}{u-\frac{c}{2} \omega_{r}}-\delta \approx \beta+\frac{b \omega_{r}}{u}-\delta
\end{array}\right.
$$

According to (2), (3), (4), the 2DOF state space equation of rear wheel steering of three wheel forklift is:

$$
\left[\begin{array}{l}
\dot{\beta} \\
\dot{\omega}_{r}
\end{array}\right]=\left[\begin{array}{ll}
a_{11} & a_{12} \\
a_{21} & a_{22}
\end{array}\right]\left[\begin{array}{l}
\beta \\
\omega_{r}
\end{array}\right]+\left[\begin{array}{l}
b_{1} \\
b_{2}
\end{array}\right] \delta
$$

Where:

$$
\begin{aligned}
& a_{11}=\frac{-\left(C_{1}+C_{2}+C_{3}\right)}{M u}, a_{12}=\frac{a C_{1}+a C_{2}-b C_{3}}{M u^{2}}-1 \\
& a_{21}=\frac{a C_{1}+a C_{2}-b C_{3}}{J_{x}}, a_{22}=-\frac{a^{2} C_{1}+a^{2} C_{2}+b^{2} C_{3}}{J_{z} u} \\
& b_{1}=\frac{C_{3}}{M u}, \quad b_{2}=\frac{b C_{3}}{J_{x}} .
\end{aligned}
$$

\subsection{Dynamics Model of Steering Executive Mechanism for SBW System}

The dynamics model of steering handle (i.e. the steering handle of forklift) can be described by the following equation:

$$
J_{h} \ddot{\theta}_{h}+B_{h} \dot{\theta}_{h}+C_{h} \theta_{h}=\tau_{h}-\tau_{f}
$$

Where, $J_{h}, B_{h}$ and $C_{h}$ are the moment of inertia of steering handle, the viscous friction coefficient and the torsional stiffness of steering shaft respectively; $\theta_{h}$ is the angle of steering handle, $\tau_{h}$ is the input hand torque of driver. $\tau_{f}$ is the feedback resistance moment which transfers to the steering shaft through the road feeling motor and deceleration mechanism.

Due to the SBW system eliminates the mechanical connection between steering handle and steering wheels, it is not necessary to consider the influence of road impact on steering system. The road feeling is totally produced by the simulation of road feeling motor [4-6]. In order to simplify the calculation, $\tau_{f}$ is designed as:

$$
\tau_{f}=\tau_{e} /\left(r_{p} \cdot G_{1}\right)
$$

Where, $\tau_{e}$ is the self-aligning torque of rear wheel, $r_{p}$ is the transmission ratio of steering gear, $G_{1}$ is the 
reduction ratio of deceleration mechanism of road feeling motor.

The actual torque is generated by steering motor, and the rear wheel is rotated by steering gear. The model of steering motor is described by the following secondorder equation:

$$
J_{s m} \ddot{\theta}_{s m}+B_{s m} \dot{\theta}_{s m}+\tau_{12}=\tau_{s m}
$$

Where, $J_{s m}$ and $B_{s m}$ are the moment of inertia and the viscous friction coefficient of steering motor respectively, $\theta_{s m}$ is the axial angle of steering motor, $\tau_{12}$ is the torque which the steering rear wheel applies to the motor shaft through the steering gear, $\tau_{s m}$ is the torque control input of steering motor, i.e. the electromagnetic torque of motor.

The steering rear wheel is used as driving load of steering motor, which rotates around the vertical axis through the wheel center. Therefore, the rotation of the steering rear wheel satisfies the following kinetic equation:

$$
J_{n} \ddot{\delta}+B_{n w} \dot{\delta}+\tau_{e}=\tau_{s}
$$

Where, $J_{r w}$ and $B_{r w}$ are the moment of inertia and the viscous friction coefficient of steering rear wheel respectively, $\tau_{s}$ is the torque exerted by the steering motor on the steering shaft through the steering gear.

Where, the relationship of $\theta_{s m}, \delta$ is:

$$
\frac{\theta_{s m}}{\delta}=\frac{\dot{\theta}_{s m}}{\dot{\delta}}=\frac{\ddot{\theta}_{s m}}{\vec{\delta}}=G_{2}=\frac{\tau_{s}}{\tau_{12}}
$$

$G_{2}$ is the transmission ratio of the steering motor to the steering shaft through the deceleration mechanism.

Linking (8), (9) and (10), it can be obtained that:

$$
J_{e q} \ddot{\delta}+B_{e q} \dot{\delta}+\tau_{e}=\tau_{e q}
$$

Where,

$$
\begin{gathered}
J_{e q}=J_{n w}+G_{2}{ }^{2} J_{s w} \\
B_{c q}=B_{n w}+G_{2}{ }^{2} B_{s m}
\end{gathered}
$$

Then the equivalent driving torque of steering electromotor is:

$$
\tau_{s q}=G_{2} \tau_{s m}
$$

It can be seen from (11) that the SBW system from the steering motor to the steering rear wheel is equivalent to a second-order direct drive system. Although the moments of inertia $J_{s m}$ and $J_{r w}$, and the viscous friction coefficient $B_{s m}$ and $B_{r w}$ are unknown, they have the following bounded conditions: $0 \leq J_{r w 0} \leq J_{r w} \leq J_{r w 1}, \quad 0 \leq B_{r w} \leq B_{r w 1}, \quad 0 \leq B_{s m} \leq B_{s m 1}$, $0 \leq J_{s m 0} \leq J_{s m} \leq J_{s m 1}$.

Besides the uncertainties above, another significant amount of uncertainty in the executive mechanism of steering system is self-aligning torque $\tau_{e}$ in the steering rear wheel, which is generated by the lateral force that suffered by the rear wheel when the forklift is steering[2-4].

$$
\tau_{e}=\left(l_{m}+l_{p}\right) F_{y 3}
$$

In the (15), $l_{p}$ is the pneumatic trail, $l_{m}$ is mechanical trail. Combining (3) and (4), the total self-aligning torque of the tire is:

$$
\tau_{e}=-C_{3} \cdot\left(l_{m}+l_{p}\right) \cdot\left(\beta+\frac{b \omega_{r}}{u}-\delta\right)
$$

For the design of sliding mode controller in the next section, the upper bound on the self-aligning torque $\tau_{e}$ of tire is estimated as follows:

$$
\left|\tau_{e}\right| \leq \bar{\tau}_{e}
$$

Where, $\quad \bar{\tau}_{e}=\bar{C}_{3} \cdot\left(\bar{l}_{m}+\bar{l}_{p}\right) \cdot\left|\beta+\frac{b \omega_{r}}{u}-\delta\right|, \bar{l}_{m}$ and $\bar{l}_{p}$ are the upper bound of $l_{p}$ and $l_{m}, \bar{C}_{3}$ is the upper bound of $C_{3}$.

\section{Steady State Control of Steering Gain for Three Wheeled Forklift}

\subsection{Description of Steering Characteristic}

When the three wheeled forklift is steering, the ratio of the steering handle angle $\theta_{h}$ to the rear wheel angle of the forklift $\delta$ is defined as the steering ratio $i[7,8]$,

$$
i=\theta_{h} / \delta
$$

By changing the transmission ratio, the SBW makes the steering characteristics exhibit a fixed proportional 
relationship, which is independent of the vehicle speed, In this paper, the ideal transmission ratio of SBW for forklift is determined based on yaw rate gain invariant $[7$, 8].

\subsection{Design of Ideal Transmission Ratio for Three Wheeled Forklift}

After a step angle input, 2DOF vehicle model of rear wheel steering three wheeled forklift achieves steady state which is the constant-speed circumference. At steady state, the yaw rate $\omega_{r}$ is constant, so $\dot{v}=0, \dot{\omega}_{r}=0, C_{1}=C_{2}$, linking (2) and (5), we can get the yaw rate as follows:

$$
\omega_{r}=\frac{2 u / L}{\frac{M u^{2}}{L^{2}}\left(\frac{2 a}{C_{3}}-\frac{b}{C_{1}}\right)+2} \delta=\frac{2 u / L}{K u^{2}+2} \delta
$$

In (19), $K\left(\mathrm{~s}^{2} / \mathrm{m}^{2}\right)$ is defined as the stability factor of three wheeled forklift, $K=M\left(2 a / C_{3}-b / C_{1}\right) / L^{2}$.

Therefore, the desired yaw rate gain $K_{s}\left(\mathrm{~s}^{-1}\right)$ is:

$$
K_{s}=\frac{\omega_{r}}{\theta_{h}}=\frac{2 u / L}{K u^{2}+2} \cdot \frac{\delta}{\theta_{h}}
$$

Then, basing on the premise that the desired yaw rate gain remains constant, the ideal transmission ratio of the three wheeled forklift is shown as follow:

$$
i=\frac{\theta_{h}}{\delta}=\frac{2 u / L}{K u^{2}+2} \cdot \frac{1}{K_{s}}
$$

\subsection{Determination of Ideal Transmission Ratio for Three Wheeled Forklift}

In order to ensure that forklift increases the steering sensitivity at low speed, increases the stability at high speed, and to ensure the yaw rate remains constant for the steering handle angle in the steering process, the change of ideal transmission ratio of three wheeled forklift with vehicle speed is designed as followed.

When the speed is below the set value $u_{0}$, the transmission ratio of the SBW system is fixed; thus the ideal transmission ratio $i$ of steering system is set as follow:

$$
i= \begin{cases}i_{\min }=1 & \left(u \leq u_{0}\right) \\ \frac{2 u / L}{K u^{2}+2} \cdot \frac{1}{K_{s}} & \left(u>u_{0}\right)\end{cases}
$$

When $i$ is fixed, the transition speed $u_{0}$ can be obtained according to (21):

$$
u_{0}=\frac{1 \pm \sqrt{1-2 i_{\min }{ }^{2} K_{s}{ }^{2} L^{2} K}}{i L K_{s} K}
$$

There is no standardized method for the selection of the yaw rate gain value up to now. According to the research and statistics of modern car: the yaw rate gain value of car is $0.16 \sim 0.33 \mathrm{~s}^{-1}[7,8]$. In this paper, four values which meet the steering characteristics for forklift are chosen as: $0.21,0.22,0.23,0.24 \mathrm{~s}^{-1}$, these values are used as the quantitative analysis, the corresponding transition speeds are $0.40,0.42,0.44,0.46 \mathrm{~m} / \mathrm{s}$ respectively. Through the above method, curves of the ideal transmission ratio with the vehicle speed are shown in Fig.2.

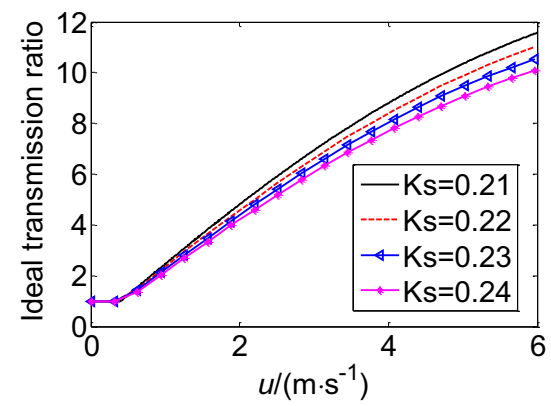

Figure 2. Variation curves of ideal transmission ratio with vehicle speed under different $K_{s}$

\section{Design of Sliding Mode Controller for Steering Executive mechanism}

The uncertain parameters in (11) makes the establishment of accurate mathematical model becomes difficult, or even destroys the tracking performance of system[9]. For the bounded information of uncertain parameters existing in (11) and the self-aligning torque in (16), the sliding mode controller is designed in this section, which makes tracking error of steering executive mechanism converge to zero gradually.

\subsection{The Mathematical Model of Steering Executive Mechanism Control System}

According to the bounded information of uncertain parameters $\tau_{e}, J_{e q}$ and $B_{e q}$, let:

$$
\begin{aligned}
& P_{a}=-\frac{B_{e q}}{J_{e q}} P_{b}=\frac{1}{J_{e q}}, P_{d}=-\frac{\tau_{e}}{J_{e q}}, P_{a 0}=-\frac{B_{e q 1}}{J_{e q 0}+J_{e q 1}}, \\
& P_{b 0}=\frac{2}{J_{e q 0}+J_{e q 1}}, \Delta P_{a}=P_{a}-P_{a 0}, \Delta P_{b}=P_{b}-P_{b 0}, \Delta P_{d}=P_{d} ;
\end{aligned}
$$

and the (11) can be rewritten as: 


$$
\ddot{\delta}=P_{a 0} \dot{\delta}+P_{b 0} \tau_{c q}+\Delta P_{a} \dot{\delta}+\Delta P_{b} \tau_{c q}+\Delta P_{d}
$$

And:

$\left|\Delta P_{a}\right| \leq \zeta,\left|\Delta P_{b} / P_{b 0}\right| \leq \xi,\left|\Delta P_{d}\right| \leq \eta,(\xi+1)(2 \xi+1)^{-1} \leq P_{b} / P_{b 0}$.

Where, $\zeta=\frac{B_{e q 1} J_{e q 1}}{J_{e q 0}\left(J_{e q 0}+J_{e q 1}\right)}, \xi=\frac{J_{e q 1}-J_{e q 0}}{2 J_{e q 0}}, \quad \eta=\frac{\bar{\tau}_{e}}{J_{e q 0}}$.

\subsection{Design of Sliding Mode Control Law}

Let $\delta_{d}$ as the target rear wheel angle, the tracking error of rear wheel angle is $e=\delta-\delta_{d}$; then it can be obtained that $\dot{e}=\dot{\delta}-\dot{\delta}_{d}, \ddot{e}=\ddot{\delta}-\ddot{\delta}_{d}$

Using the law of sliding mode control, the switching function is defined as $s=\dot{e}+\lambda \boldsymbol{e}$, where $\lambda>0$. The law of control is defined as $\tau_{e q}=\tau_{e q 0}+\tau_{e q 1}$, where $\tau_{e q 0}$ is the equivalent control, the purpose is to keep the state of the system staying on the sliding mode hyperplane. $\tau_{e q 1}$ is used to overcome the influence of uncertainties and make the system state meet the reachability condition of sliding mode. Taking the derivative $S$ with respect to time, it can be obtained that $\dot{S}=\ddot{e}+\lambda \dot{e}=\ddot{\delta}-\ddot{\delta}_{d}+\lambda\left(\dot{\delta}-\dot{\delta}_{d}\right)$; taking (24) into the above equation.

$$
\begin{aligned}
\dot{s}= & \left(P_{a 0} \dot{\delta}+P_{b 0} \tau_{e q}+\Delta P_{a} \dot{\delta}+\Delta P_{b} \tau_{e q}+\Delta P_{d}\right) \\
& -\ddot{\delta}_{d}+\lambda\left(\dot{\delta}-\dot{\delta}_{d}\right)
\end{aligned}
$$

Taking the equivalent control:

$$
\tau_{e q 0}=-P_{b 0}{ }^{-1} \mu^{\prime}
$$

Where, $\mu^{\prime}=P_{s 0} \dot{\delta}-\ddot{\delta}_{d}+\lambda\left(\dot{\delta}-\dot{\delta}_{d}\right) ;$ In order to make the system state meet the reachability condition of sliding mode, the sliding mode control is designed as follow:

$$
\tau_{\text {eql } 1}=-P_{b 0}{ }^{-1} k \operatorname{sgn}(s)
$$

Where

$k=(2 \xi+1)(\xi+1)^{-1}\left[\zeta \dot{\delta}+\eta+\xi\left|\mu^{\prime}\right|+\varepsilon\right] ; \varepsilon>0$, and it is a selectable constant.

\subsection{Proof of Stability}

Constructing Lyapunov function as $V=s^{2} / 2$, differentiating $V$ respect to time, and taking into account (25), (26) and (27), it can be obtained that:

$$
\begin{aligned}
\dot{V} & =s \cdot \dot{s} \\
& =s \cdot\left[P_{b} \tau_{e q 1}+\Delta P_{a} \dot{\delta}+\Delta P_{b} \tau_{e q 0}+\Delta P_{d}\right] \\
& =s \cdot\left[-P_{b} P_{b 0}{ }^{-1} k \operatorname{sgn}(s)+\Delta P_{a} \dot{\delta}-\Delta P_{b} P_{b 0}{ }^{-1} \mu^{\prime}+\Delta P_{d}\right] \\
& \leq-P_{b} P_{b 0}{ }^{-1} k \cdot|s|+|s| \cdot\left|\Delta P_{a} \dot{\delta}-\Delta P_{b} P_{b 0}{ }^{-1} \mu^{\prime}+\Delta P_{d}\right| \\
& \leq|s| \cdot\left[-P_{b} P_{b 0}{ }^{-1} k+\left|\Delta P_{a}\right| \dot{\delta}+\left|\Delta P_{b} P_{b 0}{ }^{-1}\right| \cdot\left|\mu^{\prime}\right|+\left|\Delta P_{d}\right|\right] \\
& \leq|s| \cdot\left[-(\xi+1)(2 \xi+1)^{-1} k+\zeta \cdot \dot{\delta}+\xi \cdot\left|\mu^{\prime}\right|+\eta\right] \\
& =-\varepsilon \cdot|s|
\end{aligned}
$$

Due to $\varepsilon>0$, it can be obtained that $\dot{V}<0(|s| \neq 0)$, the sliding mode is asymptotically reachable and the system is stable. The tracking error between the actual rear wheel angle and the target rear wheel angle can converge to zero asymptotically.

\subsection{Suppression of Buffeting}

Because of the symbol function $\operatorname{sgn}(s)$ in (27), sliding mode control in the switching action of the switching surface causes control discontinuity and result in chattering. For this problem, the saturated function $\operatorname{sat}(s)$ is used instead of $\operatorname{sgn}(s)$ in the sliding mode controller, to ensure the smooth continuity of the controlled input in the vicinity of the switching surface. Saturated function sat(s) is:

$$
\operatorname{sat}(s)= \begin{cases}\operatorname{sgn}(\mathrm{s}) & |s|>\phi \\ s / \phi & |s| \leq \phi\end{cases}
$$

Where, $\phi$ is the boundary layer.

\section{Simulation and Analysis}

\subsection{Simulation Environment}

The design parameters of SBW system are shown in Table 1, parameters of three wheeled forklift dynamic model are shown in Table 2. In the simulation test, boundary conditions of uncertain parameters in SBW system are set as follows:

$0.015 \mathrm{~kg} \cdot \mathrm{m}^{2} \leq J_{s m} \leq 0.028 \mathrm{~kg} \cdot \mathrm{m}^{2}, 0<B_{s m} \leq 0.045 \mathrm{Nms} / \mathrm{rad}$ $0<B_{r w} \leq 0.5 \mathrm{Nms} / \mathrm{rad}, 0.0040 \mathrm{~kg} \cdot \mathrm{m}^{2} \leq J_{r w} \leq 0.0048 \mathrm{~kg} \cdot \mathrm{m}^{2}$

$\bar{l}_{m}=0.02 \mathrm{~m}, \bar{l}_{p}=0.03 \mathrm{~m}, \bar{C}_{3}=160000 \mathrm{~N} / \mathrm{rad}$.

Table.1 Nominal Parameters Of SBW System 


\begin{tabular}{|c|c|}
\hline parameters & values \\
\hline$J_{h}, C_{h}\left(\mathrm{~kg} \cdot \mathrm{m}^{2}\right)$ & $0.0791,0.2$ \\
\hline$B_{h}(\mathrm{Nms} / \mathrm{rad})$ & 0.15 \\
\hline$G_{1}, G_{2}$ & $16.5,30$ \\
\hline$K_{s}\left(\mathrm{~s}^{-1}\right)$ & 0.23 \\
\hline$r_{p}, \phi$ & $20,0.05$ \\
\hline$\lambda, \varepsilon$ & $1,0.01$ \\
\hline
\end{tabular}

Table.2 Parameters of Three Wheeled Forklift Dynamics

\begin{tabular}{|c|c|}
\hline Parameters & Values \\
\hline$M(\mathrm{~kg})$ & 2937 \\
\hline$a, b, c(\mathrm{~m})$ & $\begin{array}{c}1.408,0.512, \\
1.88\end{array}$ \\
\hline$L(\mathrm{~m})$ & 1.92 \\
\hline $\begin{array}{c}\text { Wet asphalt pavement } \\
\left(C_{1}=C_{2}, C_{3}(\mathrm{~N} / \mathrm{rad})\right)\end{array}$ & 43000,80000 \\
\hline $\begin{array}{c}\text { Dry asphalt } \\
\text { pavement } \\
\left(C_{1}=C_{2}, C_{3}(\mathrm{~N} / \mathrm{rad})\right)\end{array}$ & 77850,153840 \\
\hline$J_{z}\left(\mathrm{~kg} \cdot \mathrm{m}^{2}\right)$ & 50 \\
\hline$l_{m}, l_{p}(\mathrm{~m})$ & $0.016,0.023$ \\
\hline
\end{tabular}

Simulation environment is set as follows:

(1) Using two different road environments, the first $10 \mathrm{~s}$ is set to wet asphalt pavement, the second $10 \mathrm{~s}$ is set to dry asphalt pavement.

(2) The speed of forklift is: $u=10 \mathrm{~km} / h \approx 2.78 \mathrm{~m} / \mathrm{s}$.

(3) The driver input torque is set to sinusoidal continuous input to examine the stability and the tracking performance, which is $\tau_{h}=0.5 \sin (1.9 t) \mathrm{N} \cdot \mathrm{m}$.

\subsection{Simulation Results}

The $30^{\circ}$ step signal of steering handle is simulated and analyzed. The simulation results are shown in Fig.4, Fig.5.

It can be seen from Fig. 3 and Fig. 4 that when using conventional fixed transmission ratio to control, the sideslip angle and yaw rate gain change greatly with the speed of forklift, when using ideal transmission ratio to control, the yaw rate response of the forklift does not vary with vehicle speed, which is good for improving steering performance; and the sideslip angle is obviously reduced relative to the fixed transmission ratio, which makes the forklift has better steering stability.

It can be seen from Fig.5 (a) and Fig.5 (b) that, for the steering angle tracking sliding mode control of SBW system based on ideal transmission ratio, although there are uncertain parameters exist in the system, the rear wheel angle of forklift can closely follow the change of the target rear wheel angle through the sliding mode control. Although the road environment changes at $10 \mathrm{~s}$, the SBW system is still able to meet good steering performance, which shows the strong robustness of the sliding mode control to the external conditions. Fig. 5 (c) shows the motor equivalent control torque, Fig. 5 (d) shows the uncertain self-aligning torque of the wheel and its upper bound during the process of forklift moving.
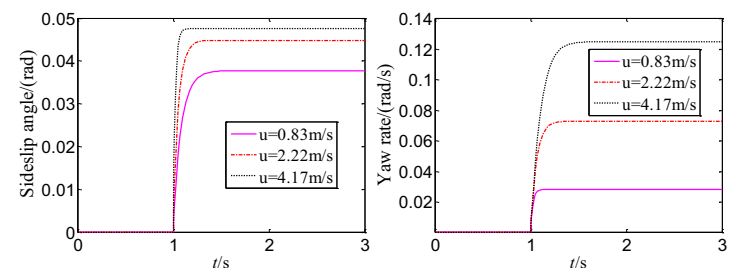

Figure 3. The step response of the steering performance when the transmission ratio is fixed to 8
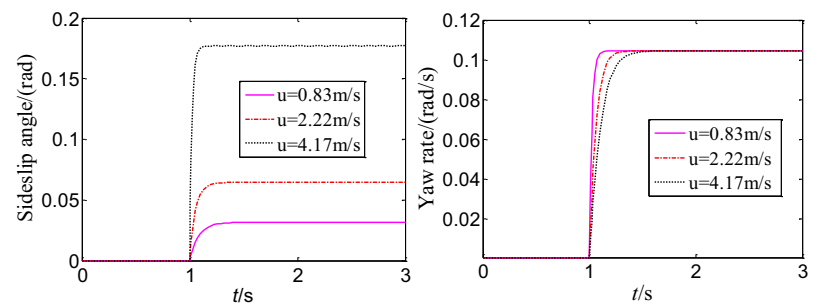

Figure 4. The step response of the steering performance under the ideal transmission ratio

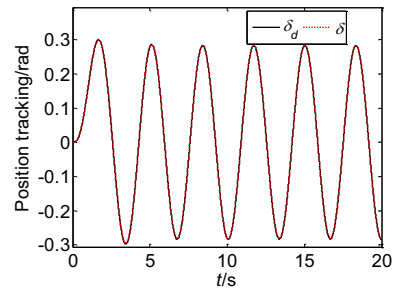

(a) Position tracking

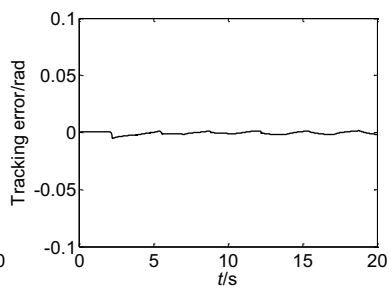

(b) Tracking error

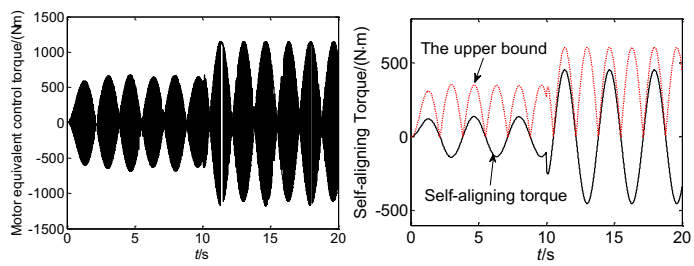

(c) Motor equivalent control torque (d) Self-aligning torque Figure 5. Sliding mode control for SBW

The simulation results of boundary layer sliding mode control is shown in Fig. 6.It can be seen from Fig. 6 (a) and Fig. 6 (b) that the SBW system exhibits good tracking performance under the boundary layer sliding mode control. Although the angle position tracking error has increased, by a reasonable choice of the thickness of the boundary layer, the position tracking error can be suppressed small enough to meet the requirements of actual operation of forklift SBW system. As shown in Fig. 6 (c), by using the boundary layer sliding mode to control, not only the chattering in the motor drive torque is effectively restrained, but also the amplitude of the equivalent drive torque is greatly reduced. 


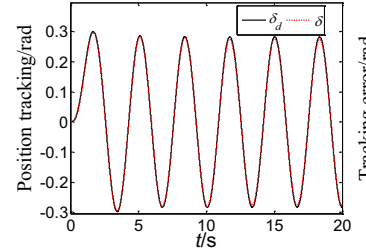

(a) Position tracking

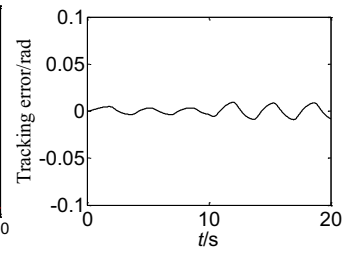

(b) Tracking error

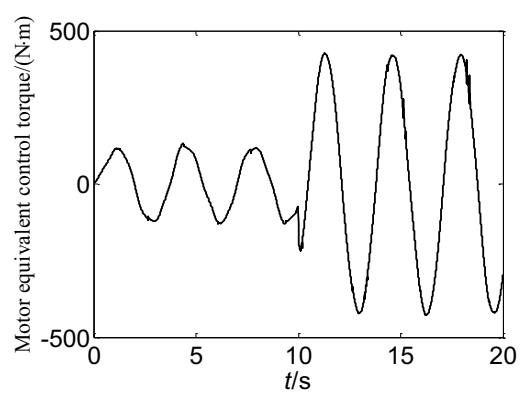

(c) Motor equivalent control torque

Figure 6. The boundary layer sliding mode control for SBW

\section{Conclusions}

Based on the structural characteristics of SBW system for three wheeled forklift, the 2DOF dynamic model and steering executive mechanism dynamic model of SBW system are deduced. A steering control strategy combining ideal variable transmission ratio and sliding mode control algorithm is proposed. The simulation results show that the steering stability of forklift can be improved by using the steering control strategy. The strategy is robust to parameter uncertainties, which makes the tracking errors converge quickly to achieve satisfactory tracking performance.

\section{Acknowledgment}

This work was supported by the National Natural Science Foundation of China under Grant No.51577046, the State Key Program of National Natural Science Foundation of China under Grant No.51637004, the national key research and development plan "important scientific instruments and equipment development" Grant No.2016YFF0102200.

\section{References}

[1] X. Lei, D. Zhang, Y. Jin, "Research on vibration suppression of the Forklift Steering System based on finite element modal analysis and pole placement," Robotics and Manufacturing Automation (ROMA), 2014 IEEE International Symposium on. IEEE Press, 2014,pp. 46-51.

[2] B. Zheng, S. Anwar, "Yaw stability control of a steer-by-wire equipped vehicle via active front wheel steering," Mechatronics, vol.19, 2009, pp. 799-804.

[3] N. Matsunaga, J. Im, S. Kawaji, "Control of steering-by-wire system of electric vehicle using bilateral control designed by passivity approach," Journal of System Design and Dynamics, vol.4, 2010, pp. 50-60.

[4] H. Wang, H. Kong, Z. Man, Z. Cao, DM Tuan, "Sliding mode control for steer-by-wire systems with AC motors in road vehicles," IEEE transactions on Industrial Electronics, vol 61, 2014, pp. 1596-1611.

[5] D. Bernardiniy, S. Di Cairanoz, A. Bemporad, H. Tsengz, "Drive-by-wire vehicle stabilization and yaw regulation: A hybrid model predictive control design," in Proceedings of the 48th IEEE Conference on. IEEE Press, 2009, pp.7621-7626.

[6] A. Rodriguez-Angeles, JA. Garcia-Antonio, "Active disturbance rejection control in steering by wire haptic systems," ISA transactions, vol. 53, 2014, pp. 939-946.

[7] C. Wang, T. Cui, W. Zhao, J. Chen, "Active front wheel steering control based on ideal transmission ratio," Transactions of the Chinese Society of Agricultural Engineering, vol. 31, 2015, pp. 85-90.

[8] H. Zheng, C. Zong, C. Tian, Z.Cheng, T. Zhu, Y. Dong, et al, "Control algorithm for steer-by-wire system with ideal steering ratio, "Journal of Jilin University (Engineering and Technology Edition), vol.37, 2007,pp. 1229-1235.

[9] D. Ren, S. Cui, J. Zhang, "Quasi-Sliding mode control for lane keeping with bounded and varying parameters," Transactions of Beijing Institute of Technology, vol.31, 2011, pp. 69-73. 